\section{S40 SURVIVAL IN DOMICILIARY NIV. AN OBSERVATIONAL COHORT STUDY OVER A 9-YEAR PERIOD}

T Ingle, A Creamer, A Patel, K Lee. King's College Hospital NHS Foundation Trust, London, $U K$

\subsection{6/thoraxjnl-2017-210983.46}

Introduction Long-term domiciliary NIV is increasingly used for patients with chronic respiratory failure, typically due to neuro-muscular disease, sleep-disordered breathing/obesity hypoventilation, obstructive lung disease (COPD) and restrictive lung disease. The benefit of domiciliary NIV in patients with neuro-muscular disease is well established, and recent evidence of the prognostic benefit in COPD may lead to increased use of home NIV in this group. Here, we intended to analyse and compare the indications for NIV, patient demographics, mode of NIV initiation (elective or emergency admission) and mortality in a cohort of patients newly-started on NIV over a 9 year period.

Methods A retrospective observational cohort study was performed using data collected between 2007 and 2015 in a single-centre teaching hospital. Patients newly started on domiciliary NIV were screened using electronic patient records and departmental NIV databases. Patients commenced on NIV for chronic respiratory failure were included, whilst cases under 18 years of age and in whom NIV was supplied for other indications (e.g., sputum clearance) were excluded. SPSS was used for analysis.

Results 311 cases were included, of which there was a slight majority of males (56.3\%). 50.2\% of patients were diagnosed with a neuro-muscular disorder (mean age of 61.6 years); $35.7 \%$ of as sleep-disordered breathing/obesity hypoventilation (mean age 56.4 years), $12.2 \%$ as COPD (mean age 62.7 years) and $1.9 \%$ of cases were established as restrictive lung disease (mean age 54 years). We found that $76.3 \%$ of COPD patients were set up acutely, whereas $73.7 \%$ of patients with neuromuscular disorders were established electively on NIV. In total, $58.1 \%$ of cases were elective starters; $49.4 \%$ patients commenced electively died within 12 months. Regression analysis indicated a significant effect of year on mortality $(p=0.002)$. Chi-square testing showed no association between initiation mode and gender $(\mathrm{p}=0.405)$.

Conclusions We demonstrate that domiciliary NIV use is particular high amongst neuro-muscular patients. Survival of patients on domiciliary NIV has improved in this single centre, and is associated with year of initiation, but mortality at 12 months is unchanged.

\section{S41 TRACHEOSTOMY VENTILATION IN MOTOR NEURONE DISEASE: A MULTI-CENTRE REVIEW}

${ }^{1} \mathrm{JM}$ Palmer, ${ }^{2} \mathrm{AD}$ Armstrong, ${ }^{1} \mathrm{~B}$ Kathiresan, ${ }^{3} \mathrm{MJ}$ Latham, ${ }^{4} \mathrm{R}$ Moses. ${ }^{1}$ Plymouth Hospitals NHS Trust, Plymouth, UK; ${ }^{2}$ The Newcastle upon Tyne Hospitals NHS Foundation Trust, Newcastle upon Tyne, UK; ${ }^{3}$ Leeds Teaching Hospitals NHS Trust, Leeds, UK; ${ }^{4}$ Lancashire Teaching Hospitals NHS Foundation Trust, Preston, UK

10.1136/thoraxjnl-2017-210983.47

Introduction and Objectives Little data exists regarding use of tracheostomy ventilation (TV) in patients with motor neurone disease (PwMND). NICE 2016 does not provide guidance for use of TV. Some centres offer TV as a treatment option. Data suggest TV in PwMND can prolong life and is more readily accepted by young males. It is hypothesised that starting TV in PwMND is intrusive to quality of life and leads to unacceptably, long hospital stays.

Methods 4 HMV centres obtained data by retrospective casenote review of patients set-up on TV as a consequence of MND between January 1998 and December 2016.

Results 38 patients (26 male) were included. Average age at tracheostomy was 59.3 (range 26-78). 79\% $(n=30)$ of patients had emergency tracheostomy v 21\% elective. $76 \%$ $(n=23)$ of emergencies were related to acute illness requiring intubation. $75 \%(n=6)$ of those who elected for TV wanted to live as long as possible or were struggling with continuous use of non-invasive interfaces, all of these lived with a partner or parent. $41 \%$ were managed on respiratory wards for the majority of the inpatient stay. After commencing TV, mean length of stay was 7 weeks for those admitted electively v 18 weeks as an emergency. 2 patients died in hospital. 71\% were discharged to their own home. Majority of home care was undertaken by skilled carers ( $22 \mathrm{hrs} /$ day) rather than Registered Professional (1.8 hrs/day). 3 patients were weaned, 1 successfully. Mean length of life post TV was 3.7 years (range 0-15 years), with longer life expectancy in the elective group (5.1 years). A total of $52 \%$ patients died during the timeframe. $45 \%$ of deaths were unexpected the rest expected or planned withdrawal.

Conclusion TV in PwMND could be associated with increased length of life. In keeping with published data there appears to be a high incidence of unexpected death. PwMND and TV tend to be discharged to their own home with skilled carers. Length of hospital stay for planned admission is not long as is anecdotally suggested. Further work, including detailed nationwide audit, national ventilation registry and national guidance may be helpful.

\section{New insights in bronchiectasis}

\section{S42 SEX DIFFERENCES IN REPORTED QUALITY OF LIFE IN BRONCHIECTASIS: AN ANALYSIS OF THE EMBARC REGISTRY}

${ }^{1} \mathrm{SM}$ Finch, ${ }^{1} \mathrm{~A}$ Shoemark, ${ }^{1} \mathrm{M}$ Crichton, ${ }^{2} \mathrm{M}$ Loebinger, ${ }^{3} \mathrm{E}$ Polverino, ${ }^{4} \mathrm{~S}$ Aliberti, ${ }^{5} \mathrm{P}$ Goeminne, ${ }^{6} \mathrm{M}$ Vendrell, ${ }^{7} \mathrm{~A}$ De Soyza, ${ }^{8} \mathrm{~K}$ Dimakou, ${ }^{1} \mathrm{JD}$ Chalmers. ${ }^{1}$ University of Dundee, Dundee, UK; ${ }^{2}$ Royal Brompton Hospital, London, UK; ${ }^{3}$ VHIR- Hospital Valle D'Hebron, Barcelona, Spain; ${ }^{4}$ University of Milan, Milan, Italy; ${ }^{5}$ AZ-Nikolaas Hospital, Sint-Niklaas, Belgium; ${ }^{6}$ Dr. Josep Trueta University Hospital, Girona, Spain; ${ }^{7}$ Newcastle University, Newcastle upon Tyne, UK; ${ }^{8}$ Sotiria Chest Hospital, Athens, Greece

\subsection{6/thoraxjnl-2017-210983.48}

Introduction Bronchiectasis is a chronic disease with a major impact on Quality of Life (QoL). As part of the EMBARC European Bronchiectasis Registry patients complete a Quality of Life-Bronchiectasis (QoL-B) questionnaire annually. The QoL-B has been validated and is widely used to give a comprehensive picture of the QoL across different aspects of patient's lives. Bronchiectasis is more common in females but sex differences in disease impact have not been explored.

Methods The EMBARC registry is a prospective observational study of adult patients with clinically significant bronchiectasis from 27 European countries. Baseline QoL-B questionnaires were analysed cross-sectionally using multiple linear regression to identify independent determinants of QoL across the 8 domains. 
Results 8389 patients were included, 58\% were female, median age 68 years. Men and women appear to have a similar level of disease severity with no significant differences in the Respiratory Symptoms, Role Functioning and Health Perception domains of the QoL-B. There was however a significant difference in all other aspects of the QoL scores. Male patients had higher scores for Physical functioning, with $\beta$ coefficient 4.9 (95\% CI 3.3-6.5, p<0.0001) (indicating an average of 4.9 points higher for men when adjusted for confounding variables). Men also had higher scores for Emotional Functioning ( $\beta$ coefficient 3.6 (95\%CI 2.2-5.1), $p<0.0001$ ), Social Functioning ( $\beta$ coefficient 2.4 (95\% CI $0.7-4.1)$, $\mathrm{p}=0.007)$ and Vitality ( $\beta$ coefficient 2.5 (95\%CI $1.2-3.9$ ), $\mathrm{p}<0.0001)$. Across all domains, the elements with a large effect were Breathlessness, Comorbidities (with Depression and Anxiety commonly having a large impact), Pseudomonas aeruginosa infection and frequent exacerbations. Female patients nevertheless had greater disease impact even after adjusting for these variables.

Conclusions From this large dataset we show that despite similar levels of disease severity, women living with bronchiectasis are significantly more affected in physical, emotional and social functioning aspects of their lives than their male counterparts. This has implications for tailoring care and suggests a need for more holistic care for patients with bronchiectasis.

\section{S43 IN-HOSPITAL MORTALITY AND LENGTH OF STAY FOLLOWING CARDIOVASCULAR EVENTS AND INTERVENTIONS IN PEOPLE WITH BRONCHIECTASIS: A POPULATION BASED STUDY}

${ }^{1} \mathrm{M}$ Plowright, ${ }^{2} \mathrm{JP}$ Hutchinson, ${ }^{3} \mathrm{JK}$ Quint, ${ }^{2} \mathrm{RB}$ Hubbard, ${ }^{2} \mathrm{~V}$ Navaratnam. ${ }^{1}$ Nottingham University Medical School, University of Nottingham, Nottingham, UK; ${ }^{2}$ Division of Epidemiology and Public Health, University of Nottingham, Nottingham, UK; ${ }^{3}$ Department of Respiratory Epidemiology, Occupational Medicine and Public Health, National Heart and Lung Institute, Imperial College London, London, UK

\subsection{6/thoraxjnl-2017-210983.49}

Background The incidence and prevalence of bronchiectasis is increasing. Epidemiological studies have reported that people with bronchiectasis are at increased risk of cardiovascular comorbidities. However, there are limited data on outcomes after acute myocardial infarction (AMI), coronary artery bypass graft (CABG) or percutaneous angioplasty (PCI) amongst people with bronchiectasis. The aims of our study were to determine in-hospital mortality and mean length of hospital stay (LOHS) following AMI, CABG and PCI in people with and without bronchiectasis.

Methods We used data from the Nationwide Inpatient Sample, an anonymised stratified yearly sample of discharge records from community hospitals in the USA developed for the Healthcare Cost and Utilisation Project. People with a record of bronchiectasis from 2000 to 2011 were identified using the International Classification of Diseases (ICD-9-CM) codes. ICD-9-CM and procedure codes were also used to identify people with AMI, CABG and PCI. Multivariable logistic regression was used to estimate odds ratios for in-hospital mortality following AMI, CABG and PCI in people with and without bronchiectasis, adjusting for age and sex. We also compared mean LOHS following AMI, CABG and PCI between individuals with bronchiectasis and the general population.

Results We identified 101597 people with a record of bronchiectasis. The mean age of the cohort was 57.2 years (Standard Deviation 20.8) and 60.8\% were female. $2195(2.2 \%)$ individuals with bronchiectasis had an AMI, 366 (0.4\%) had undergone a CABG and $827(0.8 \%)$ underwent a PCI. In-hospital mortality amongst people with bronchiectasis following AMI, CABG and PCI was $12.5 \%, 3.6 \%$ and $2.9 \%$ respectively. After adjusting for age and sex, we found no difference in inhospital mortality following AMI, CABG or PCI in people with bronchiectasis compared to the general population (Table 1). Individuals with bronchiectasis had a longer mean LOHS following AMI, CABG and PCI (Table 1).

Conclusions Our findings suggest no difference in risk of death following AMI, CABG and PCI in people with bronchiectasis, which should be taken into account when counselling patients. However, individuals with bronchiectasis had a longer mean LOHS, which may impact healthcare resources and patient care pathways.

\section{S44 RCT EVALUATION OF THE BRONCHIECTASIS EMPOWERMENT TOOL SELF-MANAGEMENT INTERVENTION USING SELF-REPORTED QUESTIONNAIRES AND FOCUS GROUPS}

${ }^{1} \mathrm{C}$ Brockwell, ${ }^{1} \mathrm{AM}$ Wilson, ${ }^{1} \mathrm{~A}$ Clark, ${ }^{1} \mathrm{G}$ Barton, ${ }^{2} \mathrm{MC}$ Pasteur, ${ }^{3} \mathrm{R}$ Fleetcroft, ${ }^{4} \mathrm{JH}$ Hill, ${ }^{1}$ A Stöckl. 'School of Medicine and Health Sciences, University of East Anglia, Norwich, UK; ${ }^{2}$ Department of Respiratory Medicine, Norfolk and Norwich University Hospital NHS Foundation Trust, Norwich, UK; ${ }^{3}$ Acle Medical Practice, Bridewell Lane, Acle, UK; ${ }^{4}$ Norfolk Community Health and Care Trust, Norwich Community Hospital, Norwich, UK

10.1136/thoraxjnl-2017-210983.50

Abstract S43 Table 1 In-hospital mortality and mean length of hospital stay following AMI, CABG and PCI

\begin{tabular}{|c|c|c|c|c|c|c|c|}
\hline & $\begin{array}{l}\text { Number of people } \\
\text { with bronchiectasis } \\
(n=101,597)(\%)\end{array}$ & $\begin{array}{l}\text { No of people without } \\
\text { bronchiectasis } \\
(\mathrm{n}=77,666,681)(\%)\end{array}$ & $\begin{array}{l}\text { In-hospital mortality in } \\
\text { people with } \\
\text { bronchiectasis (\%) }\end{array}$ & $\begin{array}{l}\text { In-hospital mortality in } \\
\text { people without } \\
\text { bronchiectasis (\%) }\end{array}$ & $\begin{array}{l}\text { Adjusted } \\
\text { Odds Ratio } \\
\left(95 \% \mathrm{Cl}^{[i]}\right)^{*}\end{array}$ & $\begin{array}{l}\text { Mean LOHS } \\
\text { people with } \\
\text { bronchiectasis (SD) in } \\
\text { [iii] })\end{array}$ & $\begin{array}{l}\text { Mean LOHS (days) in } \\
\text { people without } \\
\text { bronchiectasis (SD) }\end{array}$ \\
\hline AMI & 2195 (2.16) & $2,423,961(3.12)$ & $12.5 \%$ & $10.1 \%$ & $\begin{array}{l}0.97(0.86- \\
1.10)\end{array}$ & $7.7(8.3)$ & $6.3(7.9)$ \\
\hline CABG & $366(0.36)$ & $732373(0.94)$ & $3.6 \%$ & $3.1 \%$ & $\begin{array}{l}0.90(0.51- \\
1.57)\end{array}$ & $11.4(8.7)$ & $9.8(8.5)$ \\
\hline $\mathrm{PCl}$ & $827(0.81)$ & $1,791,318(2.31)$ & $2.9 \%$ & $1.4 \%$ & $\begin{array}{l}1.40(0.93- \\
2.10)\end{array}$ & $6.0(8.1)$ & $3.2(4.2)$ \\
\hline
\end{tabular}

${ }^{*}$ Odds ratio adjusted for age and sex

'Confidence Interval

ii Length of Hospital Stay

iiistandard Deviation 